\title{
Fluxos de energia e desenvolvimento da cultura do abacaxizeiro ${ }^{1}$
}

\author{
Cleber B. de Souza ${ }^{2}$, Bernardo B. da Silva ${ }^{3}$, Pedro V. de Azevedo ${ }^{3}$ \& Vicente de P. R. da Silva ${ }^{3}$
}

\begin{abstract}
RESUMO
O dossel vegetativo do abacaxizeiro varia consideravelmente desde o plantio das mudas até a cobertura completa, no final do ciclo produtivo. Um experimento agrometeorológico foi desenvolvido nos tabuleiros costeiros do estado da Paraíba, com o cultivo do abacaxi, cv. Pérola, de março de 2001 a julho de 2002, com o objetivo de analisar os efeitos dos componentes do balanço de energia sobre os diversos estágios de desenvolvimento do abacaxizeiro cultivado em condições de irrigação suplementar. O método do balanço de energia, baseado na razão de Bowen, foi utilizado na obtenção dos fluxos de energia durante o período experimental. Os componentes do balanço de energia foram fortemente influenciados pelo índice de área foliar e altura de planta durante todas as fases de desenvolvimento da cultura, principalmente o fluxo de calor sensível. Os resultados também indicaram que o peso da matéria seca é totalmente independente da partição do saldo de radiação em fluxo de calor latente, fluxo de calor sensível e fluxo de calor no solo. O fluxo de calor sensível variou em função do volume de água aplicado por irrigação, no cultivo de abacaxi.
\end{abstract}

Palavras-chave: evapotranspiração, razão de Bowen, fluxo de calor latente

\section{Energy fluxes and development of pineapple crop}

\begin{abstract}
The canopy of the pineapple crop changes considerably during the growing season, from a leafless stage to a fully closed canopy at the end of the productive cycle. In order to evaluate the effects of the energy balance components on the developmental stage of the pineapple crop, a field experiment was carried out from March, 2001 to July, 2002 with pineapple crop, cv. Perola, cultivated under supplemental irrigation conditions in the State of Paraíba, Brazil. The method of energy balance based on the Bowen ratio was used to obtain energy balance components during the experimental period. The energy balance components were strongly affected by leaf area index and plant height during all developmental stages of the canopy, especially the sensible heat flux. Results showed that the dry matter weight is totally independent of the energy partitioning. The sensible heat flux varied in function of the volume of water applied in irrigation during the experimental period.
\end{abstract}

Key words: evapotranspiration, Bowen ratio, latent heat flux

\footnotetext{
1 Trabalho extraído da Tese de Doutorado do primeiro autor

2 DTR/UFPB, CEP 58220-000, Bananeiras, PB. Fone: (83) 3367-1200. E-mail: cleberbrito@ig.com.br

3 UACA/UFCG, Av. Aprígio Veloso 882, Bodocongó, CEP 58109-970, Campina Grande, PB. Fone: (83) 3310-1054. E-mail: bernardo@dca.ufcg.edu.br, pvieira@dca.ufcg.edu.br; vicente@dca.ufcg.edu.br
} 


\section{INTRODUÇÃO}

O estado da Paraíba é um dos mais importantes produtores de abacaxi (Ananas comosus L.) do mundo, devido à alta produtividade e à boa qualidade dos frutos. As condições propícias de clima e solo permitem que o cultivo comercial das variedades Pérola e Smooth Cayenne se destaquem quanto aos aspectos qualitativo e quantitativo. Em condições de cultivo irrigado ou em condições de agricultura de sequeiro, muitos produtores dos tabuleiros costeiros do estado da Paraíba têm procurado inovações tecnológicas para este cultivo, com vistas ao aprimoramento do seu processo produtivo (Barreiro Neto et al., 1998).

São poucas as referências acerca do balanço de energia com o cultivo do abacaxi, apesar da sua utilização na identificação do consumo hídrico de culturas. Embora o abacaxizeiro seja cultivado em condições predominantemente de sequeiro, alguns produtores no Nordeste brasileiro têm utilizado a irrigação para garantir maior rendimento e dirigir a sua colheita para épocas que coincidam com a entressafra de sequeiro, objetivando auferir maior lucratividade. Neste sentido, o conhecimento dos componentes do balanço de energia, em particular o fluxo de calor latente, oferece elemento significativo na identificação das necessidades hídricas desta cultura.

O saldo de radiação sobre o dossel de uma cultura representa a quantidade de energia, na forma de ondas eletromagnéticas, a ser utilizada nos processos evapotranspirativo, de aquecimento do ar e do solo e na fotossíntese (Tubelis et al., 1976). Nesta concepção de análise agrometeorológica, voltada para os diversos cultivos e, especialmente, para o de abacaxi, o conhecimento da evapotranspiração ou fluxo de calor latente (LE), se torna uma ferramenta imprescindível no manejo da água, do solo e das culturas, para que se obtenha, então, sucesso na atividade agrícola.

O método do balanço de energia, baseado na razão de Bowen, constitui-se em um modelo simples e prático de estimativa do fluxo de calor latente e que tem sido amplamente utilizado com este propósito, por vários pesquisadores (Fuchs \& Tanner, 1970; Campbell, 1972; Angus \& Watts, 1984; Crago \& Brutsaert, 1996; Moran et al., 1996; Pereira et al., 1997; Perez et al., 1999; Asseng \& Hsiao, 2000); este método também é utilizado na determinação das necessidades hídricas de frutíferas cultivadas nas condições semi-áridas do Nordeste do Brasil (Azevedo et al., 2003) e no estudo dos componentes do balanço de energia da mangueira (Silva et al., 2006).

Aplicando o método do balanço de energia sobre o cultivo do abacaxi, cultivar Smooth cayenne, em Okinawa, Japão, Shiroma (1973) obteve os seguintes valores sobre a distribuição dos fluxos de energia: $40 \%$ foram destinados ao fluxo de calor latente, $56 \%$ ao fluxo de calor sensível e $4 \%$ ao fluxo de calor no solo.

Apesar da importância econômica da cultura do abacaxi para as regiões com potencial edafoclimático apropriado para o seu cultivo, pesquisas relacionadas aos seus aspectos agrometeorológicos, ainda são incipientes; neste contexto, o estudo objetivou avaliar a evolução temporal dos componentes do balanço de energia e suas relações com algumas variáveis morfológicas do abacaxizeiro cultivado em condi- ções de irrigação suplementar, nas condições de clima e solo dos tabuleiros costeiros do estado da Paraíba.

\section{MATERIAL E MÉTODOS}

O experimento agrometeorológico foi realizado nos tabuleiros costeiros do estado da Paraíba, em uma fazenda com cultivo comercial de abacaxi (Latitude: $7^{\circ}$ 14' 20" S; longitude: 34 59' 28” W; e altitude: $85 \mathrm{~m}$ ), no período de 23/03/2001 a 27/06/2002. O clima da região, segundo a classificação de Köppen, é do tipo As' - quente e úmido, com período chuvoso na estação verão-outono; valores médios diários obtidos a partir de instrumentos meteorológicos instalados na área experimental, no período de 10/08/2001 a 27/06/2002, permitiram caracterizar, por fases do cultivo, as condições meteorológicas do local do experimento, em termos de temperatura do ar, umidade relativa do ar, velocidade do vento e radiação solar (Tabela 1), enquanto os valores das precipitações pluviométricas e das irrigações mensais são apresentados na Tabela 2. Durante o período experimental os valores das temperaturas mínima e máxima foram 21,3 e $32,5^{\circ} \mathrm{C}$, respectivamente, enquanto a umidade relativa do ar variou entre 50,5 e $94,3 \%$; por outro lado, a velocidade do vento variou de 1,84 a $1,98 \mathrm{~m} \mathrm{~s}^{-1}$ e a radiação solar entre 14,51 a 21,28 $\mathrm{MJ} \mathrm{m}^{-2} \mathrm{~d}^{-1}$ (Tabela 1 ). A precipitação pluvial no período foi de 1.449,4 mm e a lâmina de água aplicada por irrigação foi de $191 \mathrm{~mm}$ (Tabela 2).

Tabela 1. Valores médios diários da temperatura do ar (Ta), umidade relativa do ar (UR), velocidade do vento $(\mathrm{V})$ e radiação solar à superfície $\left(R_{s}\right)$ para cada fase do cultivo do abacaxizeiro cv. Pérola, em Santa Rita, PB, 2001/2002

\begin{tabular}{|c|c|c|c|c|c|c|c|c|}
\hline \multirow{2}{*}{ Fases do cultivo } & \multicolumn{3}{|c|}{$\mathrm{Ta}\left({ }^{\circ} \mathrm{C}\right)$} & \multicolumn{3}{|c|}{ UR (\%) } & \multirow{2}{*}{$\begin{array}{c}V \\
\left(m \mathrm{~s}^{-1}\right)\end{array}$} & \multirow{2}{*}{$\begin{array}{c}\text { Rs } \\
\left(\mathrm{MJ} \mathrm{m}^{2}\right)\end{array}$} \\
\hline & mín & máx & méd & mín & máx & méd & & \\
\hline $\begin{array}{l}\text { Desenvolvimento } \\
\text { vegetativo }\end{array}$ & 21,3 & 31,0 & 26,2 & 5 & 94,0 & 72,3 & 1,96 & 28 \\
\hline $\begin{array}{l}\text { Florescência } \\
\text { Queda de flores }\end{array}$ & 22,5 & 32,5 & 27,5 & 5 & 93,9 & 75,3 & 1,84 & 36 \\
\hline Formação do fruto & 22,2 & 30,9 & 26 , & 62,8 & 94,3 & 78,6 & 1,89 & 17,13 \\
\hline Colheita & 21,5 & 28,8 & 25,2 & 62,8 & 94,3 & 78,8 & 1,98 & 14,51 \\
\hline Ciclo total & 21,8 & 31,2 & 26,5 & 52,5 & 94,2 & 73,4 & 1,92 & 19,98 \\
\hline
\end{tabular}

Tabela 2. Valores mensais de precipitação pluvial (Pr) e irrigações (I), obtidos na área do experimento em Santa Rita, PB, para os anos de 2001 e 2002

\begin{tabular}{|c|c|c|c|c|}
\hline \multirow{2}{*}{ Meses } & \multicolumn{2}{|c|}{ Ano 2001} & \multicolumn{2}{|c|}{ Ano 2002} \\
\hline & $\operatorname{Pr}(\mathrm{mm})$ & I (mm) & $\operatorname{Pr}(\mathrm{mm})$ & I (mm) \\
\hline Janeiro & - & - & 123,2 & - \\
\hline Fevereiro & - & - & 114,1 & - \\
\hline Março & 37,3 & - & 169,3 & 14,5 \\
\hline Abril & 167,90 & - & 48,1 & - \\
\hline Maio & 23,30 & - & 129,8 & - \\
\hline Junho & 113 & - & 256,3 & - \\
\hline Julho & 107 & - & - & - \\
\hline Agosto & 35,4 & - & - & - \\
\hline Setembro & 35,2 & 30,6 & - & - \\
\hline Outubro & 15,9 & 71,5 & - & - \\
\hline Novembro & 24,8 & 46,3 & - & - \\
\hline Dezembro & 48,8 & 28,1 & - & - \\
\hline
\end{tabular}


O solo da área experimental é do tipo Neossolo Regolítico, com textura franco-arenosa, boa drenagem e $\mathrm{pH}=4,35$ (EMBRAPA, 1999); este tipo de solo é aceitável ao cultivo do abacaxizeiro, sem nenhuma correção (Souza, 1999) razão por que se dispensou a correção do solo. Detalhes sobre os tratos culturais e fitossanitários, fertilização, irrigação e delineamento experimental desta pesquisa, podem ser obtidos em Souza et al. (2007).

A área foliar do abacaxizeiro foi obtida com um integrador de área foliar (modelo LI-3000 da LI-COR) e a altura da planta medida periodicamente. Seguindo os procedimentos estabelecidos por Silva et al. (2000) obtiveram-se os seguintes índices fisiológicos: Taxa de Crescimento Absoluto (TCA), Taxa de Crescimento Relativo (TCR) e Taxa de Assimilação Líquida (TAL).

O plantio das mudas ocorreu em 23/03/2001; o período experimental se estendeu por 450 dias após o plantio (DAP), até 15/06/2002; as variáveis morfológicas foram avaliadas 15 vezes durante o período experimental, precisamente nos 56, 84, 112, 140, 168, 196, 224, 252, 280, 308, 336, 364, 392, 420 e 448 DAP, enquanto o ciclo de desenvolvimento vegetativo do abacaxizeiro resultou nos seguintes estágios fenológicos: (I) inicial (23/03 a 18/05/2001), (II) crescimento vegetativo (19/05/2001 a 25/01/2002), (III) florescimento (26/ 01 a 08/03/2002) (obtido a partir de indução floral, à base de carbureto de cálcio (granulado: 0,5 a 1 g planta-1 $^{-1}$ colocado na roseta foliar das plantas, via funil, no horário entre 5 e 9 h (tempo local); (IV) queda de flores (09/03 a 04/04/ 2002) e (V) formação do fruto (05/04 a 18/06/2002).

No centro da área experimental (90 x 90 m), localizada numa área de produção de aproximadamente 15 ha, com espaçamento entre plantas de 0,80 x 0,25 m (50.000 plantas $\mathrm{ha}^{-1}$ ), montou-se uma mini-torre micrometeorológica, na qual foram instalados anemômetros e psicrômetros à base de termopares de cobre-constantan; os conjuntos de psicrômetros utilizados para as medições das temperaturas das termojunções seca e úmida, e de anemômetros, para a medição da velocidade do vento, foram instalados em dois níveis, separados $1,2 \mathrm{~m}$, sendo o primeiro nível tangente ao dossel vegetativo da cultura, ajustados ao longo do seu ciclo de desenvolvimento. Foram instaladas, também, duas placas de fluxo de calor no solo entre plantas colocadas em série, na profundidade de 0,02 m, a uma distância entre elas de $1 \mathrm{~m}$. Todos esses instrumentos foram alimentados por um painel solar posicionado a 2,2 m de altura. Dentro de um abrigo meteorológico instalou-se uma sonda para medições da temperatura e umidade relativa do ar. Na sistemática das observações, os dados dos instrumentos acoplados à minitorre micrometeorológica foram coletados por um sistema automático de aquisição de dados (Micrologger CR 10X), acoplado a uma placa multiplexadora (AM 416) alimentado por painel solar e programado para efetuar leituras a cada $5 \mathrm{~s}$ e armazenar médias a cada $20 \mathrm{~min}$.

As avaliações dos componentes do balanço de energia foram efetuadas considerando-se apenas os gradientes verticais, uma vez que o "fetch" utilizado (1:100) possibilitou a eliminação de eventuais efeitos de advecção. Os termos de armazenamento de calor sensível pela copa e a fração de energia utilizada na síntese biológica, foram desprezados por serem da ordem 2\% do saldo de radiação (Heilman et al., 1994); assim, a equação geral do balanço de energia foi descrita como (Azevedo et al., 2003):

$$
\mathrm{Rn}-\mathrm{LE}-\mathrm{H}-\mathrm{G}=0
$$

em que Rn é o saldo de radiação, LE o fluxo de calor latente, $\mathrm{H}$ o fluxo de calor sensível do ar e $\mathrm{G}$ o fluxo de calor no solo, todos em $\mathrm{W} \mathrm{m}^{-2}$. A razão de Bowen, $\beta$, foi estimada segundo a expressão (Rosenberg et al., 1983):

$$
\beta=\frac{H}{L E}=\frac{\mathrm{P}_{\mathrm{o}} \mathrm{C}_{\mathrm{p}}}{\mathrm{L} \varepsilon}\left(\frac{\mathrm{Kh}}{\mathrm{K} v}\right) \frac{\delta \mathrm{T} / \delta \mathrm{Z}}{\delta \mathrm{e} / \delta \mathrm{z}}=\gamma\left(\frac{\mathrm{Kh}}{\mathrm{K} v}\right) \frac{\delta \mathrm{T} / \delta \mathrm{Z}}{\delta \mathrm{e} / \delta \mathrm{Z}}
$$

em que Kh e Kv são os coeficientes de difusão turbulenta de calor sensível e vapor d'água, respectivamente, $\varepsilon=0,622$ e $\gamma$ é o fator psicrométrico $\left(\mathrm{KPa}^{\circ} \mathrm{C}^{-1}\right)$, obtido por:

$$
\gamma=\mathrm{P}_{\mathrm{o}} \mathrm{C}_{\mathrm{p}} / 0,622 \mathrm{~L}
$$

em que $\mathrm{Cp}=1004 \times 10^{-6} \mathrm{MJ} \mathrm{kg}^{-1}{ }^{\circ} \mathrm{C}^{-1}$ é o calor específico do ar a pressão constante, $\mathrm{P}_{\mathrm{o}}$ a pressão atmosférica média na superfície da região e $L$ o calor latente de vaporização (MJ kg-1), obtido pela expressão (Wrigth, 1982):

$$
\mathrm{L}=2,501-\left(2,36 \times 10^{-3}\right) \mathrm{T}_{\mathrm{a}}
$$

em que $\mathrm{T}_{\mathrm{a}}$ é a temperatura do ar $\left({ }^{\circ} \mathrm{C}\right)$. Considerando-se $\mathrm{Kh}=\mathrm{Kv}$ e

$$
\left(\frac{\delta \mathrm{T}}{\delta \mathrm{Z}}\right) /\left(\frac{\delta \mathrm{e}}{\delta \mathrm{Z}}\right) \approx \frac{\Delta \mathrm{T}}{\Delta \mathrm{e}}
$$

e sendo $\Delta \mathrm{T}=\mathrm{T}_{2}-\mathrm{T}_{1}$ e $\Delta \mathrm{e}=\mathrm{e}_{2}-\mathrm{e}_{1}$, as variações verticais de temperatura do ar e pressão de vapor na camada de ar acima do dossel da cultura, respectivamente, a Eq. 2 se torna igual a:

$$
\beta=\gamma \frac{\Delta \mathrm{T}}{\Delta \mathrm{e}}
$$

A pressão parcial de vapor d’água (e) foi calculada pela equação de Ferrel, qual seja:

$$
e\left(T_{a}\right)=e_{s}\left(T_{u}\right)-00066\left(1+0,00115 t_{u}\right)\left(T_{a}-T_{u}\right) P_{o}
$$

em que $\mathrm{T}_{\mathrm{u}}$ é a temperatura do bulbo úmido, $\mathrm{T}_{\mathrm{a}}$ é a temperatura do bulbo seco, $\mathrm{P}_{\mathrm{o}}$ é a pressão atmosférica ( $\mathrm{hPa}$ ) e $\mathrm{e}_{\mathrm{s}}\left(\mathrm{T}_{\mathrm{a}}\right)$ a pressão de saturação do vapor d'água $(\mathrm{hPa})$, que foi calculada pela equação de Tetens (Rosenberg et al., 1983):

$$
e_{s}\left(T_{u}\right)=6,1078 \exp \left(\frac{17,269 T_{u}}{237,3+T_{u}}\right)
$$

Com base na razão de Bowen, obteve-se o fluxo de calor latente segundo a expressão:

$$
\mathrm{LE}=-\left(\frac{\mathrm{Rn}-\mathrm{G}}{1+\beta}\right)
$$


O fluxo de calor sensível foi obtido como resíduo da Eq. 1, ou seja:

$$
\mathrm{H}=\mathrm{Rn}-\mathrm{LE}-\mathrm{G}
$$

Obtiveram-se as densidades de fluxos de energia para o período diurno $(\mathrm{Rn}>0)$, considerando-se positivos aqueles que chegam e negativos os que saem do sistema solo-cultura-atmosfera.

\section{RESULTADOS E DISCUSSÃO}

Os valores médios das variáveis morfológicas do abacaxizeiro em todas as suas fases de desenvolvimento, desde o plantio até a colheita, compreendidos entre 23/03/2001 a 18/ 06/2002, são apresentados na Tabela 3. O peso da matéria seca (PMS) aumentou da fase inicial (23/03 a 18/05/2001) até a fase de formação do fruto (05/04 a 18/06/2002), enquanto a altura da planta ATP estabilizou seu crescimento ao final da fase II. Por outro lado, a taxa de crescimento relativo (TCR) atinge o seu máximo na primeira fase e permanece constante nas demais, com valor mínimo de $0,01 \mathrm{~g} \mathrm{~d}^{-1}$; já a taxa de crescimento absoluto (TCA) decresceu acentuadamente desde a primeira até a última fase; a taxa de assimilação líquida (TAL), por sua vez, foi positiva apenas na primeira e última fases, permanecendo igual a aproximadamente $-0,01 \mathrm{~g} \mathrm{~cm}^{-2} \mathrm{~d}^{-1}$, nas demais fases do período analisado. O índice de área foliar (IAF) foi mínimo na fase inicial $\left(0,4 \mathrm{~m}^{2} \mathrm{~m}^{-2}\right)$ e máximo na fase de florescência $\left(9,6 \mathrm{~m}^{2} \mathrm{~m}^{-2}\right)$. Apesar do valor médio do PMS na fase de formação de frutos ter sido 650,2 g, o peso da matéria seca do abacaxizeiro de algumas plantas chegou a alcançar 831,8 g no final desta fase, precisamente aos 448 dias após plantio $(13 / 06 / 2002)$.

Tabela 3. Valores médios das variáveis morfológicos* do abacaxizeiro nas fases: (I) inicial (23/03 a 18/05/2001); (II) crescimento vegetativo (19/05/2001 a 25/01/2002); (III) florescência (26/01 a 08/03/2002); (IV) queda de flores (09/03 a 04/04/2002) e (V) formação do fruto (05/04 a 18/06/2002)

\begin{tabular}{|c|c|c|c|c|c|c|}
\hline Fases & $\begin{array}{c}\text { PMS } \\
\text { (g) }\end{array}$ & $\begin{array}{l}\text { ATP } \\
(\mathrm{cm})\end{array}$ & $\begin{array}{c}\text { IAF } \\
\left(\mathrm{m}^{2} \mathrm{~m}^{-2}\right)\end{array}$ & $\begin{array}{c}\text { TCR } \\
\left(\mathrm{g} \mathrm{d}^{-1}\right)\end{array}$ & $\begin{array}{c}\text { TCA } \\
\left(\mathrm{g} \mathrm{d}^{-1}\right)\end{array}$ & $\begin{array}{c}\mathrm{TAL} \\
\left(\mathrm{g} \mathrm{cm}^{-2} \mathrm{~d}^{-1}\right)\end{array}$ \\
\hline I & 39,64 & 9,8 & 0,4 & 0,06 & 0,53 & 0,07 \\
\hline II & 181,1 & 62,4 & 4,8 & 0,01 & $-13,37$ & $-0,02$ \\
\hline III & 417,7 & 115,3 & 9,6 & 0,01 & $-51,70$ & $-0,01$ \\
\hline IV & 419,1 & 115,3 & 9,1 & 0,01 & $-58,26$ & $-0,01$ \\
\hline V & 650,2 & 115,3 & 7,9 & 0,01 & $-152,72$ & 0,75 \\
\hline
\end{tabular}

* PMS = Peso da matéria seca; ATP = altura da planta; IAF = índice de área foliar; TCR = taxa de crescimento relativo; TCA $=$ taxa de crescimento absoluto e TAL $=$ taxa de assimilação líquida

Como as medições do balanço de energia foram iniciadas apenas a partir de 140 DAP (9/08/201), não se apresentaram os valores médios do saldo de radiação $(\mathrm{Rn})$ nem sua repartição na forma de fluxo de calor latente (LE), fluxo de calor sensível (H) e fluxo de calor no solo (G), para a fase inicial do abacaxizeiro (Tabela 4). Os valores médios de Rn, LE, H e G durante o período analisado, foram 13,44; 10,01; 2,42 e
0,91 $\mathrm{MJ} \mathrm{m}^{-2} \mathrm{~d}^{-1}$, respectivamente; por outro lado, as taxas da partição do saldo de radiação na forma de calor latente (LE/ $\mathrm{Rn})$, calor sensível (H/Rn) e calor no solo (G/Rn) foram, respectivamente, 74,95; 18,38 e 6,68\%. Valores similares da partição do saldo de radiação foram obtidos por Silva et al. (2006), em experimento com a mangueira irrigada na região submédia do rio São Francisco. As densidades de fluxos de Rn, LE e G decresceram ao longo do ciclo produtivo do abacaxizeiro; entretanto, o $\mathrm{H}$ apresentou comportamento bastante variável, enquanto a redução de Rn está associada à disponibilidade de energia na região, que foi maior no início e menor no final do período experimental, impactando diretamente o valor de LE.

Tabela 4. Valores médios dos componentes do balanço de energia ( $R n$, $\mathrm{LE}, \mathrm{H}$ e G) e do consumo do saldo de radiação (Rn) na forma calor latente (LE/Rn), fluxo de calor sensível para o ar (H/Rn) e fluxo de calor sensível para o solo (G/Rn) do abacaxizeiro nas fases: (I) inicial (23/03 a 18/05/ 2001); (II) crescimento vegetativo (19/05/2001 a 25/01/2002); (III) florescência (26/01 a 08/03/2002); (IV) queda de flores (09/03 a 04/04/ 2002) e (V) formação do fruto (05/04 a 18/06/2002)

\begin{tabular}{|c|c|c|c|c|c|c|c|}
\hline \multirow{2}{*}{ Fases } & $\mathbf{R n}$ & LE & $\mathrm{H}$ & $\bar{G}$ & LE/Rn & H/Rn & $\mathrm{G} / \mathbf{R n}$ \\
\hline & \multicolumn{4}{|c|}{ MJ $\left.m^{-2} d^{-1}\right)$} & \multicolumn{3}{|c|}{ (\%) } \\
\hline \multicolumn{8}{|l|}{ I } \\
\hline II & 13,88 & 10,49 & 1,79 & 1,60 & 75,58 & 12,86 & 11,55 \\
\hline III & 13,82 & 10,77 & 2,17 & 0,88 & 77,83 & 15,85 & 6,33 \\
\hline IV & 13,81 & 9,73 & 3,41 & 0,66 & 70,39 & 24,79 & 4,80 \\
\hline V & 11,84 & 9,06 & 2,30 & 0,48 & 76,00 & 20,00 & 4,04 \\
\hline Média & 13,34 & 10,01 & 2,42 & 0,91 & 74,95 & 18,38 & 6,68 \\
\hline
\end{tabular}

As variações dos valores acumulados dos componentes do balanço de energia do abacaxizeiro (Rn, LE, H e G), em função do índice de área foliar (IAF), são exibidas na Figura 1. Esses valores foram, durante todo o período experimental, 4.122,80, 3.122,58, 645,41 e 354,24 $\mathrm{MJ} \mathrm{m}^{-2}$, respectivamente. As curvas das densidades de fluxos de energia acumulada mudaram de comportamento quando o IAF atingiu o seu valor máximo $\left(10,23 \mathrm{~m}^{2} \mathrm{~m}^{-2}\right)$, ponto em que os valores acumu-

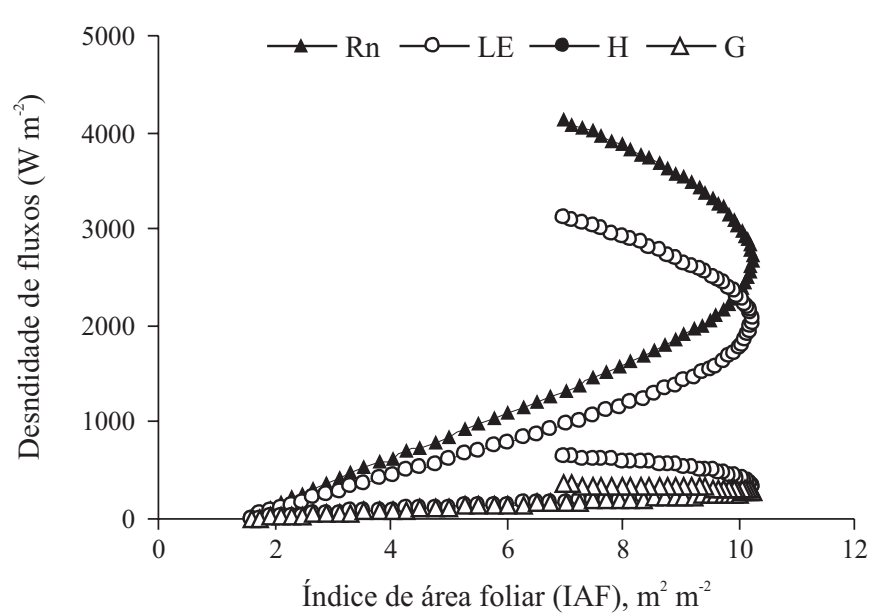

$\mathrm{Rn}=$ saldo de radiação, $\mathrm{LE}=$ fluxo de calor latente, $\mathrm{H}=$ fluxo de calor sensível e $\mathrm{G}=$ fluxo de calor no solo.

Figura 1. Variação dos valores acumulados dos componentes do balanço de energia em função do índice de área foliar (IAF) do abacaxizeiro 
lados de H e G foram rigorosamente iguais (299,59 $\left.\mathrm{MJ} \mathrm{m}^{-2}\right)$ e representaram, respectivamente, 46,4 e $84,6 \%$ do valor acumulado no período. Ainda no ponto máximo do IAF, os valores acumulados de Rn e LE corresponderam a 64,5 e 69,1\% do total acumulado no período experimental, respectivamente; portanto, apenas aproximadamente $30 \%$ do total do fluxo de calor latente foram liberados pela cultura após atingir a sua máxima área foliar.

As Figuras 2 a 4 exibem as partições do saldo de radiação (Rn) em fluxo de calor latente (LE/Rn), fluxo de calor sensível (H/LE) e fluxo de calor no solo (G/Rn), em função do índice de área foliar - IAF, altura da planta - ATP e peso da matéria seca - PMS. A taxa LE/Rn aumentou em função do IAF e ATP, enquanto o peso da matéria seca apresentou três comportamentos distintos: de 100 a 400 g de peso seco a taxa LE/Rn aumentou com o aumento do peso seco; em seguida, na faixa de 400 a 500 g, essa razão diminui e, na faixa de 500 a 800 g de peso seco, a razão volta a aumentar. A taxa LE/Rn (\%) aumentou aproximadamente 70\%, entre o IAF $=1,4$ (início das medições), a $80 \%$, para IAF $=10,2 \mathrm{~m}^{2} \mathrm{~m}^{-2}$ (Figura 2A); no entanto, há grande variabilidade associada aos valores de IAF entre 9,0 e 10,0. Similarmente, a taxa LE/Rn variou de 65 a aproximadamente $75 \%$ quando a ATP variou de 37 a $120 \mathrm{~cm}$ (Figura 2B), cujo resultado, apesar da aparente evidência, assegura que o consumo do saldo de radiação na forma de calor latente aumentou em função do aumento do dossel vegetativo da planta; já para o peso da matéria seca, flutuou de 65 a 82\%, aproximadamente; entre 100 e 400 g, sofre uma queda acentuada e volta a crescer entre 65 a $75 \%$, com o PMS de 430 a 750 g, respectivamente. Iritz \& Lindroth (1996) observaram que a taxa de evaporação (definida pela relação entre o fluxo de calor latente e o saldo de radiação) aumentou de 0,1 para 1,0 quando o IAF aumentou de 0 a $1,5 \mathrm{~m}^{2} \mathrm{~m}^{-2}$.

A Figura 3 exibe a partição do saldo de radiação em fluxo de calor sensível (H/Rn) em função de IAF, ATP e PMS. Observa-se decréscimo da taxa $\mathrm{H} / \mathrm{Rn}$, de 15 para aproximadamente $10 \%$, quando o IAF aumentou de 1,4 para $9 \mathrm{~m}^{2} \mathrm{~m}^{-2}$. A partir deste ponto, notou-se um aumento de $\mathrm{H} / \mathrm{Rn}$ até a cultura atingir a sua cobertura máxima (Figura 3A); tal inversão no comportamento na dispersão dos pontos ocorreu 275 DAP (22/12/2001). A relação entre as taxas H/Rn e ATP apresentou comportamento similar (Figura 3B). A taxa H/Rn decresceu $20 \%$, quando a ATP = $35 \mathrm{~cm}$ para $15 \%$ quando ATP $=100 \mathrm{~cm}$. Tal como no caso do IAF, a partir desse ponto a taxa H/Rn aumentou até atingir aproximadamente 30\%, quando a cultura atingiu o valor máximo (ATP $=120 \mathrm{~cm})$; neste caso, a inversão da curva ocorreu no DAP = 279 (26/ 12/2001). Durante o período experimental o total pluviométrico na região foi de $1.449,4$ mm e a irrigação aplicada, de $191 \mathrm{~mm}$ (Tabela 2); entretanto e se considerando o momento em que ocorreu a inversão das duas curvas (dias 22/12 e 26/12/2001), no período de março a dezembro, a precipitação pluvial foi de 608,6 mm (Tabela 2) e a irrigação de 176,5 mm; já no período de janeiro a junho de 2002 a precipitação e a irrigação foram de 840,8 e 14,5 mm, respectivamente; portanto, no período em que foi aplicada a maior taxa de irrigação, o consumo do saldo de radiação, na forma de calor sensível, decresceu com os aumentos do IAF e da ATP porém, no período em que se aplicou a irrigação de apenas 14,5 mm, a taxa H/Rn cresceu em função dessas variáveis morfológicas, cujos resultados sugerem que o fluxo de calor sensível é muito sensível a alterações no conteúdo de água disponível à cultura. Durante o período experimental o peso da matéria seca variou de 1,15 g $(\mathrm{DAP}=1)$ a $777,8 \mathrm{~g}$ (DAP = 450); entretanto, entre 100 e $400 \mathrm{~g}$ houve diminuição da taxa H/Rn (Figura 3C) e, nesse mesmo intervalo, ocorreu o inverso com a taxa LE/Rn, o que está muito
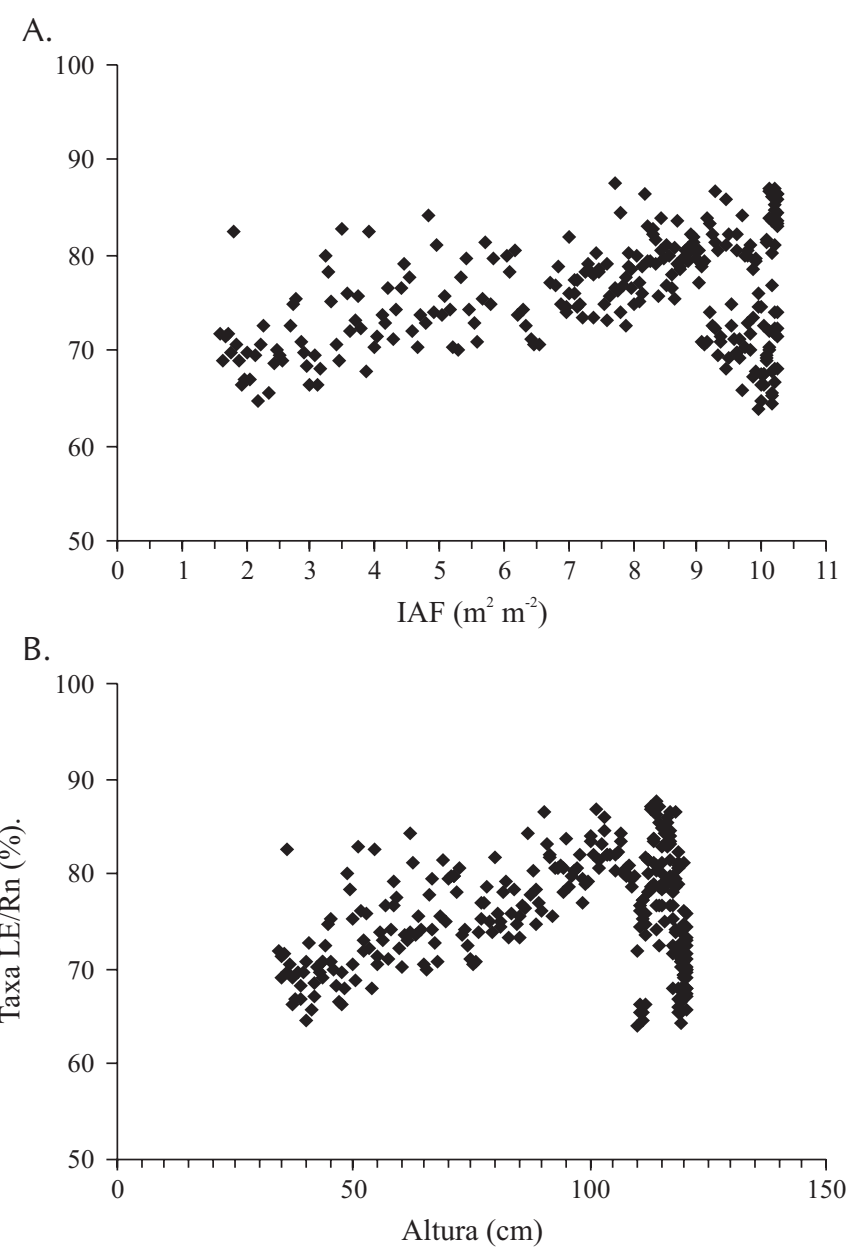

C.

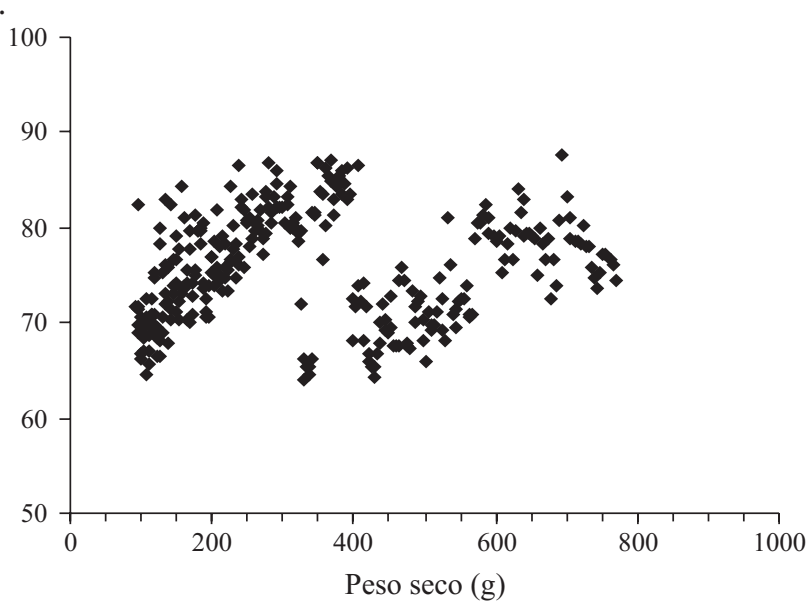

Figura 2. Partição do saldo de radiação em fluxo de calor latente (LE/Rn) em função do índice de área foliar - IAF (A), altura da planta (B) e peso da matéria seca (C) do abacaxizeiro 
concordante; ocorre, em seguida, um aumento brusco e na faixa de 430 a 770, aproximadamente, um decréscimo em $\mathrm{H} / \mathrm{Rn}$ que vai de $30 \%$ e alcança menos de $20 \%$. Este comportamento deve estar associado às flutuações no conteúdo de água do solo em que, embora as chuvas ocorram com relativa freqüência, sua ausência por um período de 5-7 dias poder alterar significativamente a disponibilidade hídrica das plantas, como resultado de uma grande oferta energética, notadamente de origem solar.

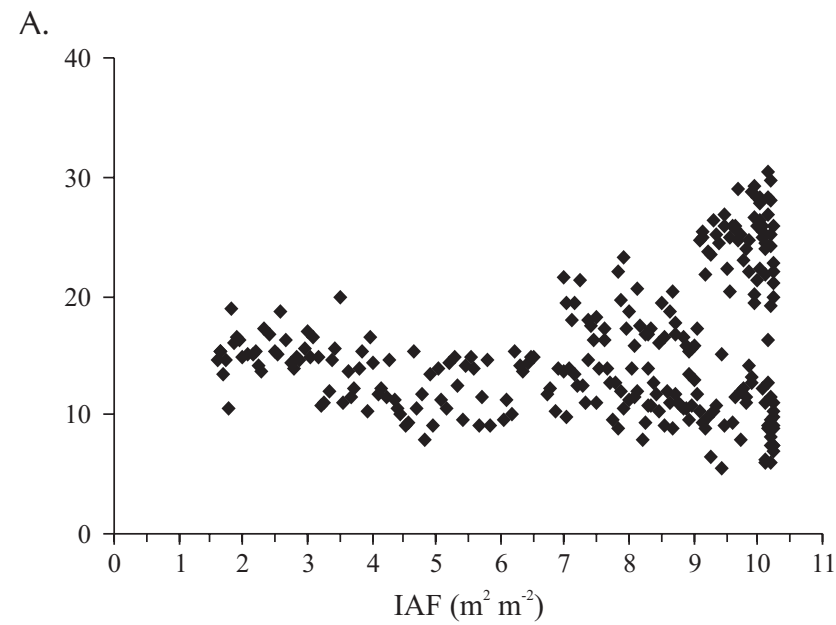

B.

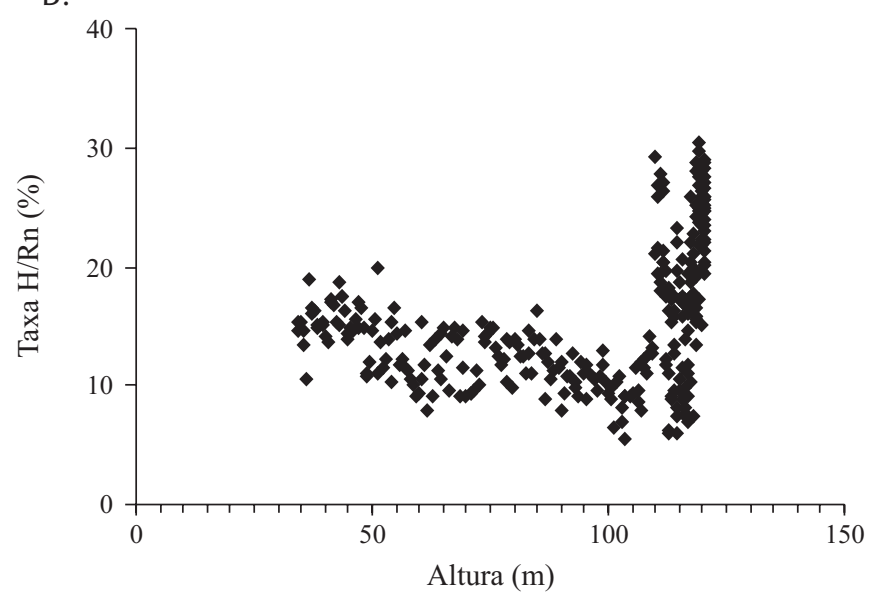

C.

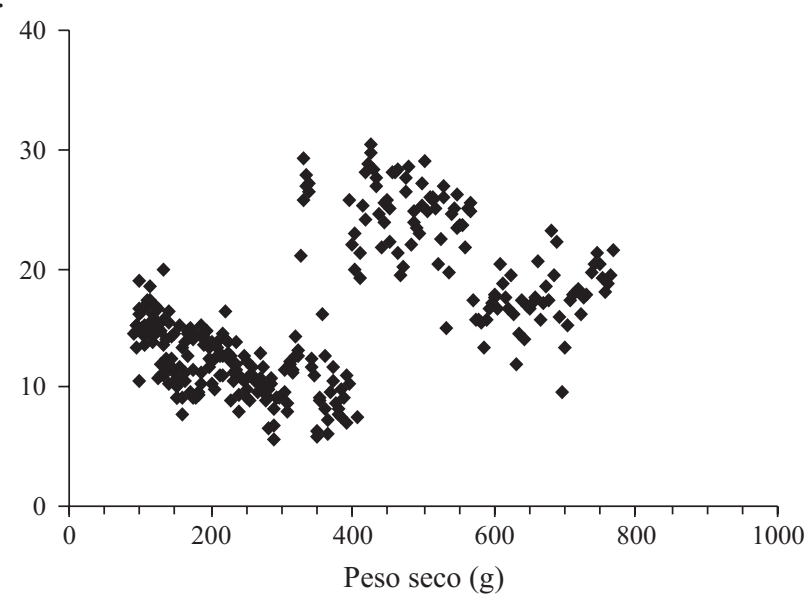

Figura 3. Partição do saldo de radiação em fluxo de calor sensível $(H / R n)$ em função do índice de área foliar - IAF (A), altura da planta (B) e peso da matéria seca $(\mathrm{C})$ do abacaxizeiro
A partição do saldo de radiação em fluxo de calor no solo (G/Rn) em função do índice de área foliar, altura da planta e peso da matéria seca do abacaxizeiro, é exibida na Figura 4, na qual se nota que a taxa G/Rn decresceu com o aumento dessas variáveis morfológicas; esta taxa decresceu de 13 para 5\%, durante o período em que o IAF variou de 1,59 a $10,47 \mathrm{~m}^{2} \mathrm{~m}^{-2}$ (Figura 4A); similarmente, a taxa G/Rn decresceu de 15 para $8 \%$ sempre que ATP variou de 35 a $120 \mathrm{~cm}$ (Figura 3B). O decréscimo do peso da matéria seca

A.

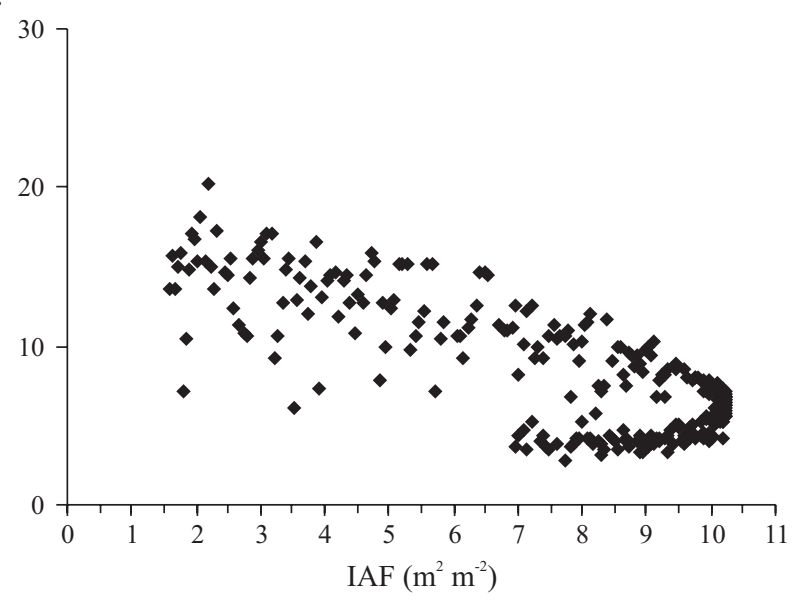

B.

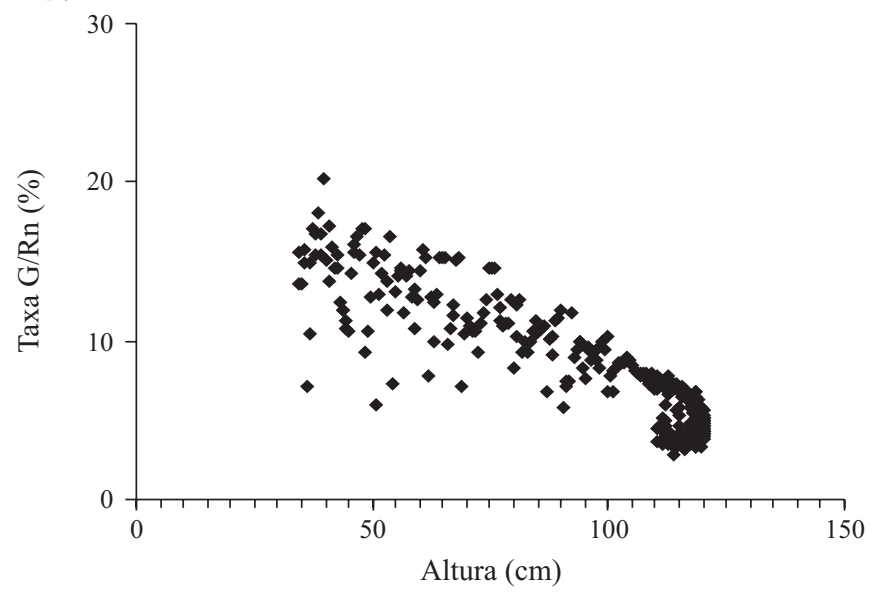

C.

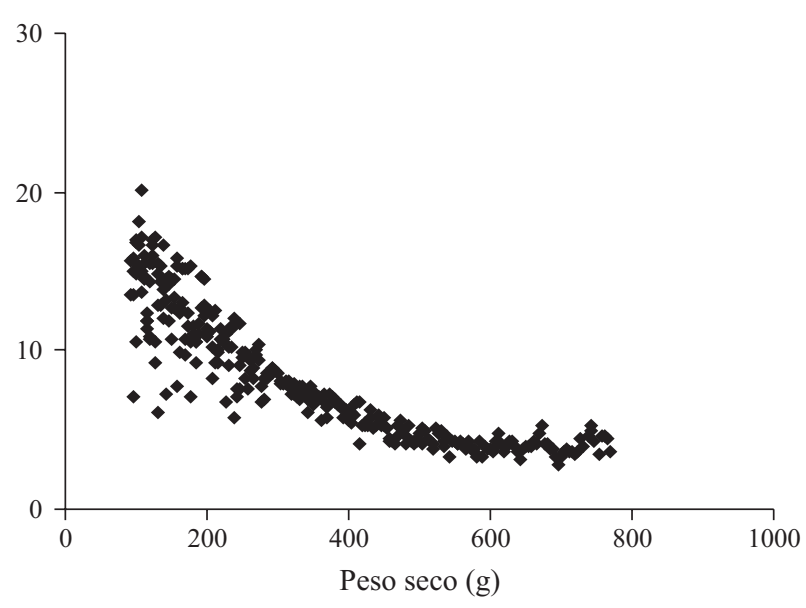

Figura 4. Partição do saldo de radiação em fluxo de calor no solo $(\mathrm{G} / \mathrm{Rn})$ em função do índice de área foliar - IAF (A), altura da planta (B) e peso da matéria seca (C) do abacaxizeiro 
em função de G/Rn foi mais acentuado; referida taxa decresceu de 15 a 5\% quando o PMS aumentou de 100 para 777,6 g (Figura 4C). O decréscimo do consumo do saldo de radiação na forma de fluxo de calor no solo, está associado às variações fisiológicas da cultura e às condições meteorológicas da área de estudo. Em estudos com IAF e taxa de evaporação em floresta, Iritz \& Lindroth (1996) obtiveram resultados similares.

Os componentes do balanço de energia para dias típicos do ciclo produtivo do abacaxizeiro com diferentes índices de área foliar, são exibidos na Figura 5. Os três dias analisados apresentaram intensa variabilidade na nebulosidade, conforme se observa no comportamento diurno dos componentes do balanço de energia, e também indicaram valores máximos em torno do meio-dia e mínimos ao nascer e pôr-dosol. O fluxo de calor no solo foi o menor dos cinco componentes do balanço de energia, em todos os dias analisados.

No dia com IAF $=7,4 \mathrm{~m}^{2} \mathrm{~m}^{-2}(19 / 11 / 2001)$ os valores máximos de Rn, LE, H e G foram 716,9, 550,5, 91,2 e 76,6 W m², respectivamente (Figura 4A). Em 06/03/2002, o dossel do abacaxizeiro atingiu a sua cobertura máxima, com IAF $=10,23 \mathrm{~m}^{2} \mathrm{~m}^{-2}$; neste dia, as densidades de fluxo de Rn, LE, H e G foram de 601,5, 347,8, 188,7 e $41,47 \mathrm{~W} \mathrm{~m}^{-2}$, respectivamente (Figura $5 \mathrm{~B}$ ); esses valores se mostraram inferiores àqueles observados no dia com IAF $=7,4 \mathrm{~m}^{2} \mathrm{~m}^{-2}$, exceto para o fluxo de calor sensível. $\mathrm{Na}$ fase final do ciclo produtivo do abacaxizeiro, quando ele se encontrava com IAF $=7,5 \mathrm{~m}^{2} \mathrm{~m}^{-2}$, os valores máximos das densidades de fluxo de Rn, LE, H e G foram 568,4, 442,3, 104,6 e 21,5 W m².

O valor máximo da densidade de fluxo de calor no solo no final do ciclo produtivo da cultura, foi o menor dentre os três dias analisados, além de apresentar valores regularmente decrescentes. As densidades de fluxo de calor latente e calor sensível, não seguiram este padrão de comportamento; portanto, o dossel vegetativo da cultura desempenha papel fundamental na moderação do fluxo de calor no solo. No dia com alta cobertura vegetativa (IAF $=10,23$ ), os fluxos de LE e H mostraram o menor e o maior valor, respectivamente, dentre os dias analisados; este resultado é aparentemente contraditório haja vista que se esperava que a cultura apresentasse a taxa máxima de evapotranspiração no período de máximo desenvolvimento vegetativo; desta forma se infere que a energia disponibilizada na forma de Rn e não utilizada pela cultura na forma de LE ou G, foi transformada em fluxo de calor sensível produzindo, portanto, o valor máximo dessa densidade de fluxo dentre os três dias analisados, em que a razão disso é atribuída às características morfofisiológicas da cultura do abacaxizeiro e ao possível déficit de umidade nesse dia; além disso, a evapotranspiração é responsável pelo processo de resfriamento das plantas; os resultados também sugerem que a densidade de fluxo de calor sensível é o componente do balanço de energia mais influenciado pela área foliar do abacaxizeiro e veranicos.

O fluxo de calor no solo o é pela área foliar e ainda por outros fatores, tais como o saldo de radiação e o conteúdo de água no solo.
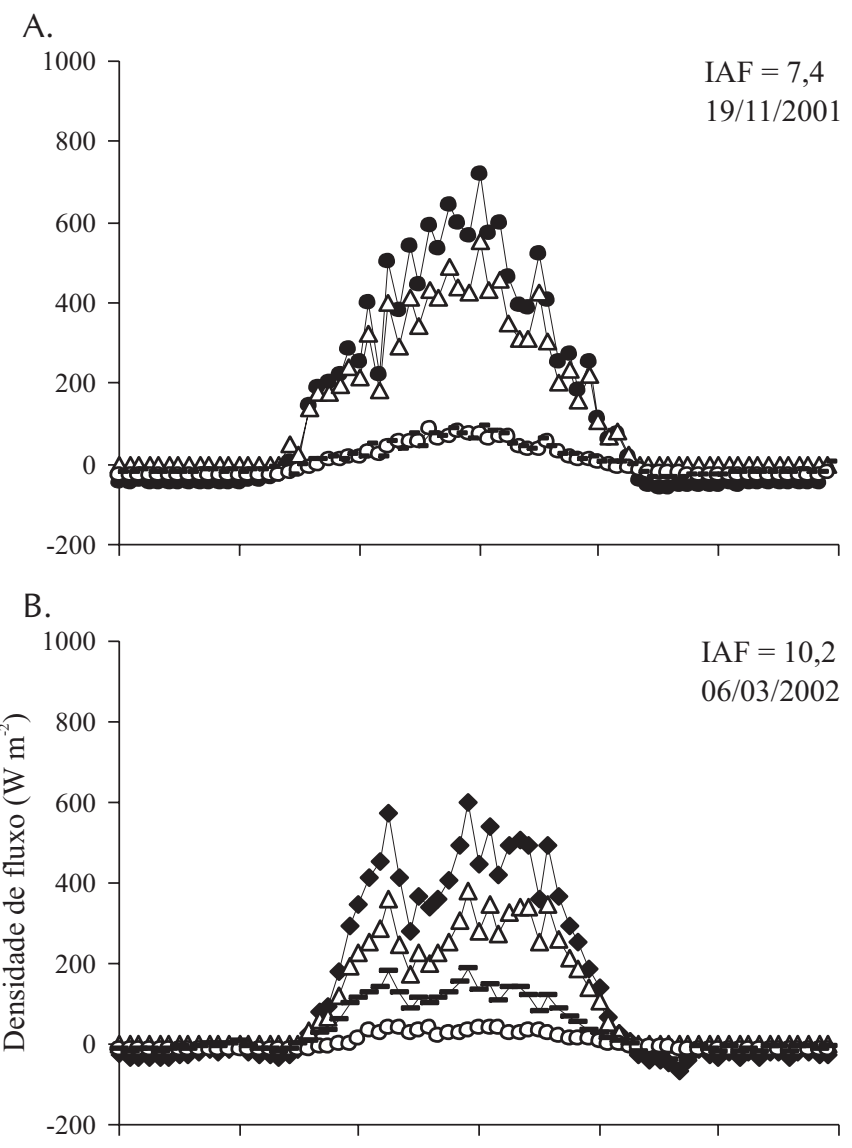

C.

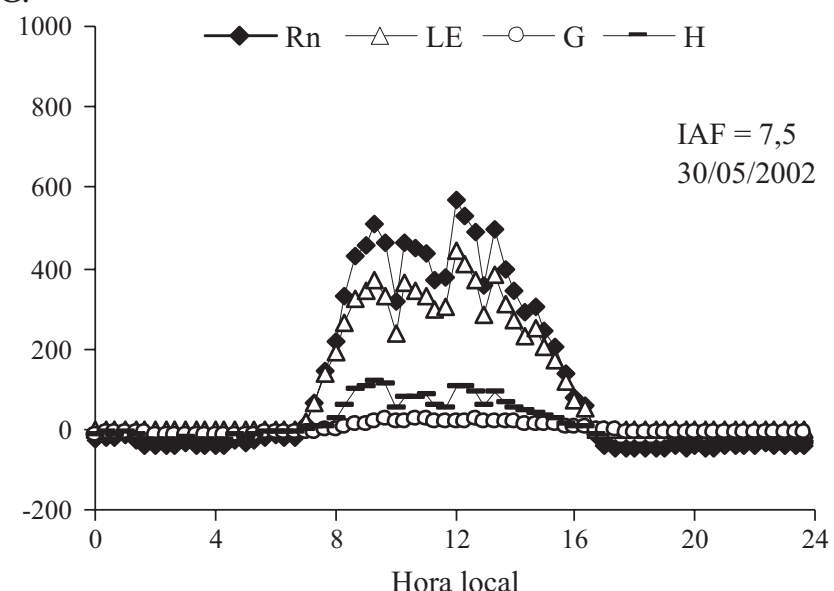

$R n=$ saldo de radiação $\left(w m^{-2}\right), L E-$ fluxo de calor latente $\left(w m^{-2}\right), H-$ fluxo de calor sensível $(w$ $\mathrm{m}^{-2}$ ) e $\mathrm{G}$ - fluxo de calor no solo $\left(\mathrm{w} \mathrm{m}^{-2}\right)$

Figura 5. Componentes do balanço de energia para dias típicos do ciclo produtivo do abacaxizeiro, com diferentes índices de área foliar (IAF)

\section{CONCLUSÕES}

1. Os componentes do balanço de energia são influenciados pelo índice de área foliar e altura da planta durante todas as fases de desenvolvimento do abacaxizeiro, principalmente o fluxo de calor sensível.

2. As partições dos componentes do balanço de energia não sofreram alterações perceptíveis com as variações no peso da matéria seca do abacaxizeiro.

3. O fluxo de calor sensível sofre forte influência do volume 
de água aplicado por irrigação, no cultivo de abacaxi.

4. O consumo do saldo de radiação na forma de calor latente aumenta em função do aumento do dossel vegetativo do abacaxizeiro.

\section{LITERATURA CITADA}

Angus, D. E.; Watts, P. J. Evapotranspiration. How good is the Bowen ratio method? Agricultural Water Management, v.8, n.1, p.133-150, 1984.

Asseng, S.; Hsiao, T. C. Canopy CO2 assimilation, energy balance, and water use efficiency of an alfalfa crop before and after cutting. Field Crops Research, v.67, n.1, p.191-206, 2000.

Azevedo, P. V.; Silva, B. B.; Silva, V. P. R. Water requirements of irrigated mango orchard in Northeast Brazil. Agricultural Water Management, v.58, n.3, p.241-254, 2003.

Azevedo, P. V.; Sousa, I. F.; Silva, B. B.; Silva, V. P. R. Wateruse efficiency of dwarf-green coconut (cocos nucifera L.) orchards in Northeast Brazil. Agricultural Water Management, v.84, n.1, p.259-264, 2006.

Barreiro Neto, M.; Choairy, S. A.; Lacerda, J. T. Caracterização do abacaxizeiro Pérola no estado da Paraíba. Pesquisa Agropecuária, EMEPA-PB, v.1, n.10, p.33-39, 1998.

Campbell, A. P. The effect of stability on the evaporation rates measurement by the energy balance. Agricultural Meteorology, v.1, n.11, p.261-267, 1972.

Crago, R.; Brutsaert, W. Daytime evaporation and the self-preservation of the evaporative fraction and the Bowen ratio. Journal of Hydrology, v.178, n.1-4, p.241-255, 1996.

EMBRAPA - Empresa Brasileira de Pesquisa Agropecuária. Centro Nacional de Pesquisa de Solos. Sistema brasileiro de classificação de solos. Brasília: Embrapa CNPS, 1999, 412p.

Fuchs, M.; Tanner, C. B. Error analysis of Bowen ratios measured by differential psichrometry. Agricultural and Forest Meteorology, v.7, n.1, p.329-334, 1970

Heilman, J. L.; McLnnes, K. J.; Savage, M. J. Soil and canopy energy in a west Texas vineyard. Agricultural and Forest Meteorology, v.71, n.1-2, p.99-114, 1994.
Iritz, Z.; Lindroth, A. Energy partitioning in relation to leaf area development of short rotation willow coppice. Agricultural and Forest Meteorology, v.81, n.1, p.119-130, 1996.

Moran, M. S.; Rahman, A. F.; Washburne, J. C. Combining the Penman-Monteith equation with measurements of surface temperature and reflectance to estimate evaporation rates of semiarid grassland. Agricultural and Forest Meteorology, v.80, n.24, p.87-109, 1996.

Pereira, A. R.; Villa Nova, N. A.; Sediyama, G. C. Evapo(transpi)ração. 1.ed. São Paulo: FEALQ, 1997. 183p.

Perez, P. J.; Castellvi, F.; Ibáñez, M;, Rosell, J. L. Assessment of reliability of Bowen ratio method for partitioning fluxes. Agricultural and Forest Meteorology, v.97, n.3, p.141-150, 1999.

Rosenberg, N. J.; Blad, B. L.; Verma, S. B. Microclimate: The biological environment. 2.ed, Lincoln - Nebraska: John Wiley \& Sons, 1983. 495p.

Shiroma, M. Evapotranspiration of pineapple in Okinawa measured by heat balance method. Science Bulletin of the College Agriculture, University of the Ryukyus, v.20, n.1, p.169-190, 1973.

Silva, L. C.; Beltrão, N. E. M.; Amorim Neto, M. Análise de crescimento de comunidades vegetais. Campina Grande: Embrapa CNPA, 2000. 46p. Circular Técnica, 34

Silva, V. P. R; Azevedo, P. V.; Silva, B. B. Seasonal patterns of the energy balance components in a mango (Mangifera indica L.) orchard grown in Northeast Brazil. Agricultural Journal, v.1, n.1, p.18-23, 2006.

Souza, C. B.; Silva, B. B.; Azevedo, P. V. Crescimento e rendimento do abacaxizeiro nas condições climáticas dos Tabuleiros Costeiros do estado da Paraíba. Revista Brasileira de Engenharia Agrícola e Ambiental, v.11, p.134-141, 2007.

Souza, L. F. S. Exigências edáficas e nutricionais. In: Cunha, G. A. P.; Cabral, J. R.; Souza, L. F. da S. O abacaxizeiro: Cultivo, agroindústria e economia. Brasília: Embrapa. Comunicação para Transferência de Tecnologia, 1999, 480p.

Tubelis, A.; Nascimento, F. J. L.; Folion, L. L. Estimativa da radiação solar global diária em Botucatu, SP, a partir da insolação diária. Pesquisa Agropecuária Brasileira, v.26, n.1, p.53-60, 1976.

Wright, J. L. New evapotranspiration crop coefficients. Journal of the Irrigation and Drainage Division, v.108, n.2, p.57-74, 1982. 